\title{
Using naturalistic driving films as a design tool for investigating driver requirements in HMI design for ADAS
}

\author{
Minjuan Wang ${ }^{*}$, Dong Sun, and Fang Chen \\ Interaction design department, Chalmers University of Technology, Sweden
}

\begin{abstract}
In recent years, there are many naturalistic driving projects have been conducted, such as the 100-Car Project (Naturalistic Driving study in United State), EuroFOT(European Large-Scale Field Operational Tests on Vehicle Systems), SeMiFOT(Sweden Michigan Naturalistic Field Operational Test and etc. However, those valuable naturalistic driving data hasn't been applied into Human-machine Interaction (HMI) design for Advanced Driver Assistance Systems (ADAS), a good HMI design for ADAS requires a deep understanding of drive environment and the interactions between the driving car and other road users in different situations. The results demonstrated the benefits of using naturalistic driving films as a mean for enhancing focus group discussion for better understanding driver's needs and traffic environment constraints. It provided an efficient tool for designers to have inside knowledge about drive and the needs for information presentation; The recommendations for how to apply this method is discussed in the paper.
\end{abstract}

Keywords: Focus group, real traffic scenarios, expert evaluation, 3D sound information system

\section{Introduction}

In recent years, Vehicles are more and more equipped with Advanced Driver Assistance Systems (ADAS). Examples of ADAS are: Lane Departure Warning (LDW), Blind Spot Information System (BSIS), Pedestrian protection system, and Adaptive Cruise Control (ACC) and etc. These systems are designed to support the driver in certain driving situations and intend to increase the driving comfort and safety. When the technical is mature, before installing it to the car, some HMI design questions need to be answered, for example, how shall the sensor information be presented to the driver? How shall we let the driver interacting with the system? What and when the information shall be presented and in what modality? All of these questions pointed to one direction, that what is the driver needs during his/her drive to avoid potential traffic accident. In order to have a deep understanding of the questions, one needs to research on how the driver interacts with other road users and traffic environment. Stoner, H.A. described that driving is a much complex domain,
" physical and intentional constraints govern the environment, events often evolve over seconds, and responses depend upon highly tuned skill-based behavior [1]. A mismatched information perception between system and driver's mental mode can lead an accident in a second. Therefore, understanding the physical and drivers intentional constraints are vital important in the ADAS HMI design. When looking at the traditional ways of ADAS design, often the design were based on predefined design guidelines, standards, use scenarios or user study results, of course, those tools are efficient and invaluable. However, it seems the design process have been taken away from it natural use contexts, and the traditional tools might not support designers to have a full comprehension of driving environmental constraints and identify drivers dynamic needs during his/her driving task. There are different ways to study the needs from the driver, work domain analysis method from Ecological interface design theory provides the way of analysis the design needs through analysis con-

\footnotetext{
* Corresponding author. Email: minw@chalmers.se
} 
straints from environment [2] [3]. Still, it is quite abstracts and the outcome of the analysis are more or less subjective.

In present study, we investigated a method of using naturalistic driving films to lead focus group discussion to discover the driver's needs. Recently, there are many naturalistic driving projects have been conducted, such as the 100-Car Project(Naturalistic Driving study in United State) [4], and similar projects like euroFOT (European Field Operational Test) [5], SeMiFOT(Sweden Michigan Naturalistic Field Operational Test) [6] and etc. Neale indicated that those projects provide vital exposure and pre-crash data for understanding causes of accidents. The applications of the naturalistic driving data have mainly focused on the evaluation of risky driving behavior and crash risk, calculation of relative risk of engaging in secondary tasks, and evaluation of driver response to traffic related information and etc[4]. Those results are invaluable for the evaluation and testing of ADAS, but we have not seen any studies which apply naturalistic driving films in the early stage of ADAS design. The driving films from the naturalistic driving database have a continuous view into the happenings in and around the vehicle; these films can provide very good resources for the designer to have an inside view of the natural driving context and the driver's behaviors and needs under different situations. In the design process, engineers normally would apply different scenarios or storyboards to build up the common understand of some particular situations [7]. These stories would contribute to a larger narrative at entire design team and project members. The real-time recorded naturalistic drive films can act as the scenarios and storyboards, the design team therefore can build up a common ground for understanding the design constraints and problems which need to take into considerations. It can also let the designers to evaluate the proposed design and its positive or negative impacts.

According to Langford, the problems or design deficiency identified by the focus group provides a tangible baseline that can lead directly to a range of solutions, at the same time, the new or modified set of user requirements might also be identified[8]. Macaulay stated that the results gained through focus group studies give the requirement engineer insights into how users think and what things are important to them[9]. Norman [10] argues that participants in focus groups have great trouble imaging how they may use new products because much of people's current behavior is not conscious, 'we are primarily aware of our acts when they go wrong or when we have diffi- culty'. The traditional focus group study often isolates people from the context where they used to perform the task and people actually use their environments and tools to prompt the sequence of actions, and focus group can be misled if the members in the group do not have the common understanding of the problem or situation. Based on Johnson \& Sutcliffe discussion, scenario-based representations and animated simulations can help users see the implications of system behavior and thereby improve validation [11] [12]. Chin states that bring scenarios based discussion into focus group studies requires participants to incorporate system features into their original usage scenarios and consider how they would be modified [13].

In present paper, we study the method of apply naturalistic driving films in the focus group discussion to discover drivers' needs. To make the study more focus, we consider the $3 \mathrm{D}$ sound presentation of traffic environment as the information presentation modality. [14] indicated that a strong positive support that the design of using natural sound to present the traffic situation to the truck drivers in 3 dimensions. It is not clear that if it is a useful way of presenting information to personal vehicle as well. At the same time, there are many design questions about what the driver needs is and what information in the traffic should to be presented, how to design the sound, should the sound information represent the location or movement of the road objects and at what time frame, etc, that one need to answer.

The focus of this paper is to not describe the details of the driver needs for the design, rather the methodology of using naturalistic driving films as a design tool for focus group studies to explore the needs from drivers. The results of this paper will assess the benefits and drawbacks of using driving naturalistic films as design tools.

\section{Method}

In this study, 4 naturalistic driving films were selected to facilitate the focus group discussion. A concept design of apply 3D sound to represent the traffic situation was proposed. Seven focus group studies have been conducted in the simulation room where the $3 \mathrm{D}$ surrounding sound system was built. The concept design, experimental design and procedure are described in the below section. 


\subsection{The concept design of $3 D$ sound traffic information system}

The proposed concept is using 3D sound to represent the traffic information in terms of road objects types, locations, movements, and distance to the personal vehicle. This exploits the possibilities of augmenting drivers hearing sense to the surrounding information. To develop the prototype of $3 \mathrm{D}$ sound traffic information system, we use Soundtrack Pro ${ }^{1}$ (see figure 1) to code sound corresponding to the road users occurred in the naturalistic driving films. There are four different types of road users are identified from the films: pedestrian, bicyclist, motorcycle, and vehicle. Different natural sounds are selected that can closely associate with the objects that the objects sound in natural environment. All sound samples are chosen from Adobe sound sample database. At the same time, another group of abstract sounds were also selected. In this group, there is not clear association of the sound and the objects it presents.

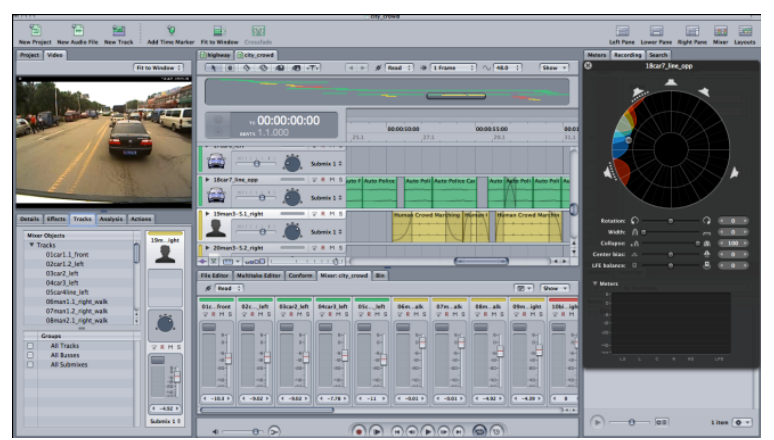

Figure 1 the interface of soundtrack pro

The proposed system presents the road users of the relative location, distance, and moving direction towards the filmed vehicle. Figure 2 illustrated how the system works.

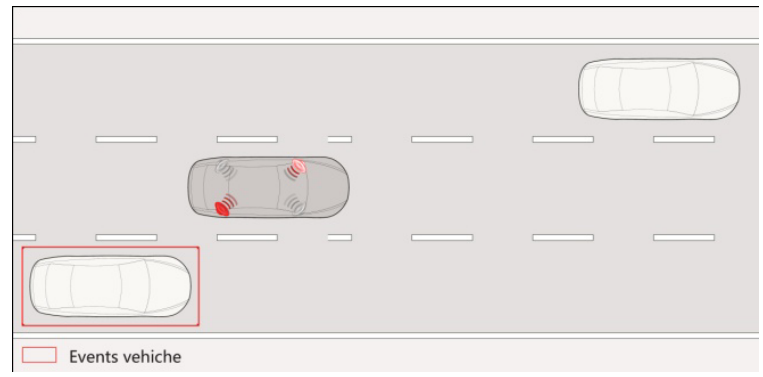

Figure 2 illstrustration of 3D sound system

\footnotetext{
${ }^{1}$ http://www.apple.com/logicstudio/soundtrackpro
}

For what traffic information should be presented with sound cues, there are 3 criterion are set: 1. only the road users are represented by the corresponding sounds, with the location, and movement transition. 2 . The road objects have interactions or potential interactions with the filmed vehicle were selected, 3 . The road signs, landmarks and infrastructure information are excluded in this study.

In each film, the proposed system will simulate different road users' movements with $3 \mathrm{D}$ sound. As Van Der Horst (15) indicated that, warning signal for Time To collision (TTC) needs to present at least $2.7 \mathrm{~s}$ in advance. As we are considering in a normal driving situation, the activation time of the sound for each object is set between 3.7 to $4 \mathrm{~s}$. We observed the road users which approaching to the experimental vehicle in the films, we activate and deactivate the sound information when the road objects are near to the experimental vehicle within the range of $4 \mathrm{~s}$ TTC.

\subsection{Experimental environment}

To evaluate the validity of the concept design, and driver's attitudes \& needs towards the system, we set up a $3.5^{*} 3.5 \mathrm{~mm}$ test environment, and it consists of a 5.1 surrounding system: 3 speakers placed at the front side and two at the rear position. The position structure of the 5.1 surrounding systems and seating position of participants are structured according to Dobly 5.1 Home theater speaker guideline ${ }^{2}$. To reduce the echo effects, noise absorbing curtains are installed on the three sides of the test area. The detail settings are showed in figure 3.

Speaker system is also one of the most important factors of hardware settings which may directly affect final outcome of the experiment. The speaker system used in this study the Logitech Z-5500.
2 www.dolby.com/consumer/setup/speaker-setupguide/index.html 


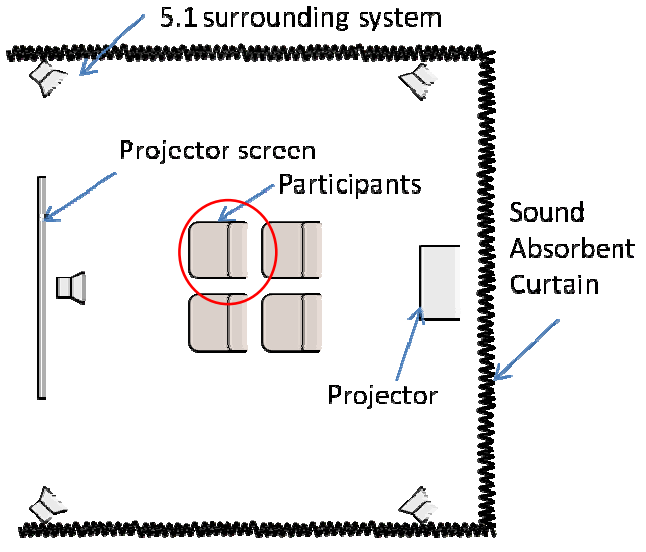

Figure 35.1 surrounding system and experimental room settings

The experimental setting intends to provide a realistic feeling of the system that the participants can experience the proposed concept design and it use context in a natural way.

\subsection{The selection of video films}

There are over thousands hours video records on naturalistic drives from both Sweden and China are available for the project. After a careful observation of the video films, 85 short video were extracted, these video clips have a combination of most frequently happened traffic incidents that occur in the video records. From 85 short videos, 4 videos from Chinese road were chosen to apply for this experiment. The reason of selecting these 4 films is that the events occurred in the video has covered most of happenings in Swedish Driving Films. These 4 videos represent 4 typical road scenarios: high way, city road, community, and roundabout. Each film has represented the unique characteristic of the scenario, for instance, speed limits, road conditions, and mixture of road participants. Table 1 showed the description of scenarios.

\begin{tabular}{|c|l|l|l|}
\hline No. & Name & Speed & Objects represented by sound \\
\hline 1 & Highway & $50 \mathrm{~km} / \mathrm{h}$ & Vehicles \\
\hline 2 & Roundabout & $30 \mathrm{~km} / \mathrm{h}$ & Vehicles, Bicycles, Motorcycles \\
\hline 3 & Community & $\begin{array}{l}\text { Less than } \\
30 \mathrm{~km} / \mathrm{h}\end{array}$ & $\begin{array}{l}\text { Pedestrians (moving and stand- } \\
\text { ing) }\end{array}$ \\
\hline 4 & City & $40 \mathrm{~km} / \mathrm{h}$ & Bicycles, Vehicles \\
\hline \multicolumn{3}{|c|}{ Table1 scenarios description }
\end{tabular}

\subsection{Experimental procedure}

The experiment includes 4 sections, the total duration of the experiment is 1.5 hours; the first part is pre-questionnaires where participants' background, 3D sound experience, driving personality is collected. In second part, each selected sound sample is played in the random order and the participants vote for the preference level of each sound sample in the ques- 
tionnaire; and a short training section is carried here, it also warm up for a further step to help participants get familiar with the 3D sound environment.

In the third part, focus group discussions are taken place, four different traffic scenarios are demonstrated with 3D sound traffic information system and the playlist of four scenarios is randomized for different discussion groups. The participants are asked to sit in the center of the test area, and experience the 3D sound traffic information first, after each play, a predefined discussion questions are asked, the questions are related in several aspects: information needs for sound display, opinions towards sound and interaction design, and deep discussion about information needs for each particular events in the scenarios. Each scenario discussion is around 15 to 20 minutes. The participants were also asked to point out the traffic information within the scenarios which they consider significant and relevant to their drive. In the end, they instruct to evaluate the pros and cons of current concept design. In the end of each discussion, participants also ask to brainstorm how should the sound be signed and other situations which they think 3D auditory information would benefit their daily drive. The whole discussions have been filmed for data analysis. The last section of the study is postquestionnaire where the suggestions for the experimental design are gathered.

\section{Results and Discussion}

The focus groups discussion were recorded and transcribed, The results of the study have generated a great number of meaningful data for the $3 \mathrm{D}$ sound traffic information system design; over 900 comments and options have been extracted from focus group transcripts, the comments have been sorted into 7 thematic categories: 1. information needs, 2 . information prioritization rules, 3 . sound design suggestions, 4. preferences and suggestions to natural and abstract sound sample design, 5. the timing for auditory notification requirements, 6 . requirements for 3D dimensional effects design, 7. different system mode suggestion. The results provided very detail information about the driver's needs for different traffic information under different drive condition and the strategies of how this information should be presented. Several system design modals can be formulated.

The results demonstrated the benefits of using naturalistic driving films as a mean for enhancing discussion for better understanding the road dynam- ics and traffic environment. It provided a useful tool for designers to have inside knowledge about drive and the needs for information presentation; it served as the basis for detailed analysis of traffic events and its interaction. The video films provides the similar functions as what [13] has described that the scenarios enable the users to immediately connect the scenarios to their own image of their work during design process; users develop a greater sense of ownership of the analysis and design. Therefore, bring the real traffic film into HMI design, it provided an opportunity that everyone involved in focus groups has the same view of the problem and situation. It helps them to understand the interaction between experimental car and other road users in real environment. Through the discussion around each event in the scenarios, members in each focus group often express their opinions and experience in their own drive and often bring up new scenarios and use case for the proposed system. Followings are some examples of comments:

"The video film gives me a clear view of what happen on the road, it remains me about the situation which I experienced this morning, when I try to change lane to the left, but I wasn't aware of the car in the later position in the left lane, I almost crash on that car, I think the system is quite useful in this kinds of situations"

(The comments from the drivers groups)

"I think the pedestrians in front of me is not interesting for me, the ones hidden behind obstacles are more important. I have been driving on this kind of road situation once; I almost had an accident, which a lady runs out from the corner, I didn't have time to react"

(A female expert in road safety domain)

"The video film is a very good way of presenting the traffic dynamics in such situation; however, the view from the video films is still not same as the real world. If you can make the display more realistic with a wider angle, it might help me to get more engaged."

(An expert in vehicle design domain)

The selection of video clips in this study is a key issue and it can strongly influence the discussion results. To perform the similar kind of study, the video films should be selected based on the research questions and interests. In the present study, we want to identify what is the driver's needs and what information do they want that would be presented through sound. Therefore, 4 different scenarios were chosen, these 4 scenarios are in different dimensions: high 
speed, low speed, high traffic density, low traffic density. These scenarios were carefully selected from the naturalistic driving database; therefore, they are representative to some common happened drive situations. Traffic situation is complicated and there are a lot of scenarios one can select. The focus group discussion through the 4 films already took 1.5 hours. From practical perspective, the focus group study is not possible to cover a lot of scenarios, before people lost the interests and focus. By comparing the data from the 4 scenarios discussion, the result can draw a brief conclusion of drivers' attitudes and needs under each scenarios.

By using naturalistic driving film together with the system prototypes in focus group discussion, the participants have the possibilities to understand the design concept correctly, and can evaluate the positive and negative sides of the design. Coughlan has also indicated in his study that offering participants something tangible to experience during a focus group session leads to a deeper understanding of concepts [15]. In the discussion, most participants commented that the study is very interesting and fun. Design expert groups admitted that the method has a high potential in the concept design phrase, and it gave more sense of reality.

"It was easy to talk about the issues within the video, but also come into other discussion and sharing some ideas, the approach is very good for initial concept design"”

(HMI designer working in car industry)

"It is a very good approach for presenting and evaluating ideas, this should be applied into industry design as well."

(Project manager for In Vehicle HMI group)

The bias of using this approach in the focus group discussion is the fidelity of the prototype will have a great impact on how the participant perceive the concept, and the design of the system can be misled the discussions. The participants would focus on criticizing the details of the design, forgot to develop the concept further with their daily drive context.

"The abstract sound design is irritating, the system is useless at all, and I would turn all the sounds off. During my drive, I will only trust my own experience and visual sense... Don't like the system at all. "

(One expert in traffic safety domain)

Some participants have also point out that
"The main problem of the method is that the prototype design is so important, what you think it very much depends on the design."

(One sound expert)

"The view of the film is too narrow, it has a smaller view that the real driving situation, the information needs can be different if the view of films are broader."

(One of the drivers )

To diminish these biases, the instructors should emphasis the purpose of the study in the very beginning of the experiment; explain the function purposes and logical of the system in a very careful way, make sure the participants understand the proposed the design is a starting point of the potential system, not the final design. And the prototype of the concept system should be more realistic, the fundamental function and information should be presented to members of focus group.

The structure of focus group is another vital issue for the study. The dominant and highly opinionated member of the group may lead the discussion to certain direction and influence the views of other members in the group. It also can be problematic with too quite or reserved participants who may be hesitant to contribute their opinion and views to the discussion. The moderator has to steer the discussion in a good way, when the discussion runs into irrelevant areas, the moderator has to intervene to bring things back on track. In some group discussion, the discussion will be lively and revealing. In others it might be slow and lethargic. Therefore, using the naturalistic films can help to reduce the effect of above problems at some extent, it help the discussions focus on the film content, and engages the participants to discuss the problems in the same domain. And each scenario discussion should not over 20 minutes long, the participants will "dry out" after one hour discussion, and the moderator should have certain strategies for steering the discussion, the members of focus group always are excited when they first enter the discussion in first scenario, so in the first scenario, the moderator should probe more deeply with discussion, and in the later scenarios, moderator can approach the same issues from several different angles to test that the responses are consistent.

\section{Conclusion}

Without a clear understanding of driver's needs and real driving context, then the designers are forced 
to "best guess" or interpret desired functionality, which inevitably leads to poor In Vehicle HMI design. Introducing naturalistic driving films into the requirement generation phase, it can encourage designers to examine how the current design fulfill driver's needs in the real situations, and force designers to think about how drivers perform the design, when they do and how they do under similar situations.

When apply Naturalistic Driving Film into the design process, there are several aspects need to take into consideration.

- The selection of the films

The films should select based on the research questions and interest. And also try to identify the interesting events in the selected films, and focus the discussion on the identified events. It will help to structure the discussion and provide a good reference for data analysis as well.

- The fidelity of the concept design is very important, which fidelity of the prototype should decided by the interests of the study. To explore user requirements and needs for the potential design, the low to medium fidelity prototype would be enough, the focus should aim on understand users needs under environmental constraints.

To evaluate the interface design, a higher fidelity prototype is needed.

- Participants selection

Choosing traffic safety and HMI experts for focus group discussion is an effective way to gather information and ideas in their specific knowledge domains; however, it's also

\section{References}

[1] Stoner, H.A., E.E. Wiese, and J.D. Lee. Applying ecological interface design to the driving domain: the results of an abstraction hierarchy analysis. 2003: Human Factors and Ergonomics Society.

[2] Ireland, C. and B. Johnson, Exploring the FUTURE in the PRESENT. Design Management Journal (Former Series), 1995. 6(2): p. 57-64.

[3] Lee, J.D., H.A. Stoner, and D. Marshall. Enhancing interaction with the driving ecology through haptic interfaces. in Systems, Man and Cybernetics, 2004 IEEE International Conference on. 2004.

[4] Neale, V.L., et al., An overview of the 100-car naturalistic study and findings. National Highway Traffic Safety Administration, Paper, 2005.

[5] Benmimoun, A., et al. euroFOT: Large scale field operational test-Impact Assessment-. 2009. risky that the result of the discussions can be very different from the real drivers.

Main advantages:

- Valuable for understanding the driving environment constraints and drivers needs

- Intuitive for the participants to understand, the participants find it creative and interesting

- The prototype help to visualize the ideas

- Provide a stimulus and common ground for discussion and probing

Main disadvantages:

- Time-consuming, it takes a large amount of time to develop the system prototype

- The design of the prototype have great influence on the discussion

Using naturalistic driving film to support In Vehicle HMI design cannot solve all the problems in the design process, it has certain drawbacks, but we believe this method can benefit the designer to have a better understanding of the design constraints and provide an efficient tool for the designers to gain a varieties of new design ideas and possibilities.

\section{Acknowledgement}

This study is conducted with EFESOS project and financed by Volvo Car Corporation and the Swedish research foundation.

[6] Karlsson, I. and P. Engelbrektsson, ON THE ISSUE OF COLLECTING SUBJECTIVE DATA: EXPERIENCES FROM A SMALL-SCALE FIELD OPERATIONAL TEST.

[7] Lloyd, P., Storytelling and the development of discourse in the engineering design process. Design Studies, 2000. 21(4): p. 357-373.

[8] Langford, J.D. and D. McDonagh, Focus groups: supporting effective product development. 2003: CRC.

[9] Macaulay, L.A., Requirements engineering. 1996: SpringerVerlag.

[10] Norman, D.A., The invisible computer. 1998: MIT press Cambridge.

[11] Johnson, W.L., M.S. Feather, and D.R. Harris, Representation and presentation of requirements knowledge. Software Engineering, IEEE Transactions on, 1992. 18(10): p. 853-869.

[12] Sutcliffe, A., User-centred requirements engineering. 2002: Springer Verlag.

[13] Chin Jr, G., M.B. Rosson, and J.M. Carroll. Participatory analysis: Shared development of requirements from scenarios. 1997: ACM. 
[14] Chen, F., G. Qvint, and J. Jarlengrip, Listen! There Are Other Road Users Close to You - Improve the Traffic Awareness of Truck Drivers. Universal Access in Human-Computer Interaction. Ambient Interaction, 2007. 4555.
[15] Coughlan, P. and A. Sklar, Bringing real world context into the focus group setting. Focus groups: supporting effective product development, 2003: p. 122. 